\title{
ADDITION TO ‘AN UPPER BOUND FOR THE NUMBER OF ODD MULTIPERFECT NUMBERS'
}

\author{
PINGZHI YUAN ${ }^{\bowtie}$ and ZHONGFENG ZHANG
}

(Received 16 January 2013; accepted 7 February 2013; first published online 7 June 2013)

\section{Abstract}

The main result in the earlier paper (by the first author) is improved as follows. The number of odd multiperfect numbers with at most $r$ distinct prime factors is bounded by $4^{r^{2}} / 2^{r+2}(r-1)$ !.

2010 Mathematics subject classification: primary 11A25.

Keywords and phrases: odd perfect numbers, $k$-perfect numbers.

\section{Introduction}

The terminology and notation in [4] are continued in this note.

In [4], the first author proved that for each positive integer $r$, the number of odd multiperfect numbers $N$ with $\omega(N) \leq r$ is bounded by $4^{r^{2}}$ when $r$ is large enough. The purpose here is to use a similar method to that in [4] to obtain the following improved estimate, valid for all $r$.

THEOREM 1.1. Let $r$ be a positive integer. The number of odd multiperfect numbers with at most $r$ distinct prime factors is bounded by $4^{r^{2}} / 2^{r+2}(r-1) !$.

Theorem 1.1 is a corollary of the following result.

THeOREM 1.2. Let $x$ and $r$ be positive integers. The number of odd multiperfect numbers $N \leq x$ with at most $r$ distinct prime factors is bounded by $\left(\begin{array}{c}\left.\log _{3} x\right\rfloor+r-1 \\ r-1\end{array}\right) 2^{r-2}$.

\section{Proofs}

Proof of Theorem 1.2. The proof is essentially a modification of the proof in [4]. Suppose that $N \leq x$ is odd $k$-perfect, $k \geq 2$ and $\omega(N) \leq r$. By a result in [1], we have $r \geq$ $k^{2}-1 \geq 3$ and $k<r$. Write $N=A B$, where $A:=\prod_{p^{e} \| N, p>2 r} p^{e}$ and $B:=\prod_{p^{e} \| N, p \leq 2 r} p^{e}$. We have

$$
\frac{\sigma(A)}{A}=\prod_{p^{e} \| A}\left(1+\frac{1}{p}+\cdots+\frac{1}{p^{e}}\right)<\prod_{p \mid A}\left(1+\frac{1}{p}+\frac{1}{p^{2}}+\cdots\right),
$$

Supported by NSF of China (No. 11271142) and the Guangdong Provincial Natural Science Foundation (No. S2012010009942).

(C) 2013 Australian Mathematical Publishing Association Inc. 0004-9727/2013 \$16.00 
and so

$$
\frac{A}{\sigma(A)}>\prod_{p \mid A}\left(1-\frac{1}{p}\right) \geq 1-\sum_{p \mid A} \frac{1}{p} \geq 1-\frac{r}{2 r+1}>\frac{1}{2} .
$$

Thus $\sigma(A)<2 A$, which implies that $B>1$. Since $N$ is $k$-perfect, $\sigma(A B)=k A B$, and hence

$$
\frac{k}{2} B<\frac{A}{\sigma(A)} k B=\sigma(B) \leq k B,
$$

with equality on the right precisely when $A=1$. Suppose that $A \neq 1$. Then, by (2.2),

$$
\sigma(B)>\frac{k B}{2} \text { and } \sigma(B) \mid k A B .
$$

If $\operatorname{gcd}(A, \sigma(B))=1$, then by the second formula of (2.3), $\sigma(B) \mid k B$, and so $\sigma(B) \leq$ $k B / 2$, which contradicts (2.3). Therefore there is a prime $p$ dividing $\operatorname{gcd}(A, \sigma(B))$, which means that $\sigma(B)$ has a prime factor $p$ with $p>2 r$ and $\operatorname{gcd}(p, B)=1$ by the definition of $A$. Let $p_{1}$ be the smallest prime divisor of $\sigma(B)$ with $p_{1}>2 r$. Then $p_{1} \mid A$ since $k<r$. Suppose that $p_{1}^{e_{1}} \| A$, where $e_{1} \geq 1$. Then if we put

$$
A^{\prime}:=A / p_{1}^{e_{1}} \quad \text { and } \quad B^{\prime}:=B p_{1}^{e_{1}},
$$

it is clear that (2.1)-(2.3) hold with $A^{\prime}$ and $B^{\prime}$ replacing $A$ and $B$. By the same argument as in [3], continuing the above procedure, we eventually obtain a factorisation

$$
A=p_{1}^{e_{1}} p_{2}^{e_{2}} \cdots \cdots p_{t}^{e_{t}},
$$

where $t=\omega(A)=\omega(N)-\omega(B) \leq r-1$.

We note that the prime $p_{1}$ depends only on $B$, while, for $i>1$, the prime $p_{i}$ depends only on $B$ and the exponents $e_{1}, \ldots, e_{i-1}$, and, moreover, $p_{t}$ and $e_{t}$ depend only on $B$ and the exponents $e_{1}, \ldots, e_{t-1}$. It follows that for a given $B$ the cofactor $A$ (if $A>1$ ) is entirely determined by $e_{1}, \ldots, e_{t-1}$, and we have $e_{i} \leq \log _{5} x$ for $i=1, \ldots, t$.

Let $B=q_{1}^{f_{1}} q_{2}^{f_{2}} \cdots q_{s}^{f_{s}}$. Then $f_{j} \leq \log _{3} x$ for $j=1, \ldots, s$, and $s+t=r$. Let $m$ be the number of odd primes not exceeding $2 r$, so $m \leq r-2$. To estimate the number of possibilities for $B$ and $e_{1}, \ldots, e_{t-1}$, we first choose $s$ odd primes $(1 \leq s \leq r-2)$ from the first $m \leq r-2$ odd primes. Then choose $f_{j} \leq \log _{3} x$ for $j=1, \ldots, s$ and $e_{i} \leq$ $\log _{5} x$ for $i=1, \ldots, t-1$ with $s+t=r$ and obviously $e_{1}+\cdots+e_{t-1}+f_{1}+\cdots+f_{s} \leq$ $\log _{3} x$. The number of possibilities for $e_{1}+\cdots+e_{t-1}+f_{1}+\cdots+f_{s} \leq \log _{3} x$ is equal to the number of nonnegative integer solutions of the equation

$$
e_{1}+\cdots+e_{t-1}+f_{1}+\cdots+f_{s}+y=\left\lfloor\log _{3} x\right\rfloor,
$$

which is $\left(\begin{array}{c}\left\lfloor\log _{3} x\right\rfloor+r-1 \\ r-1\end{array}\right)$. Therefore the number of possibilities for $B$ and $e_{1}, \ldots, e_{t}$ is bounded by

$$
\left(\begin{array}{c}
\left\lfloor\log _{3} x\right\rfloor+r-1 \\
r-1
\end{array}\right) \sum_{i=0}^{r-2}\left(\begin{array}{c}
r-2 \\
i
\end{array}\right) \leq\left(\begin{array}{c}
\left\lfloor\log _{3} x\right\rfloor+r-1 \\
r-1
\end{array}\right) 2^{r-2} .
$$

This completes the proof of Theorem 1.2. 
Proof of Theorem 1.1. By a result of Nielsen [2], we have $N<2^{4^{r}}$. By Theorem 1.2, we may take $x=2^{4^{r}}$. Then, since $4^{r} \log _{3} 2+r-1 \leq 4^{r}$, the number of odd $k$-perfect numbers $N$ with $\omega(N) \leq r$ is bounded by

$$
\left(\begin{array}{c}
\left\lfloor 4^{r} \log _{3} 2\right\rfloor+r-1 \\
r-1
\end{array}\right) 2^{r-2} \leq \frac{4^{r(r-1)} 2^{r-2}}{(r-1) !}=\frac{4^{r^{2}}}{2^{r+2}(r-1) !} .
$$

This proves the theorem.

\section{Acknowledgement}

The authors are grateful to the editor for his valuable suggestions.

\section{References}

[1] P. J. McCarthy, 'Odd perfect numbers', Scripta Math. 23 (1957), 43-47.

[2] P. Nielsen, 'An upper bound for odd perfect numbers', Integers 3 (2003), A14, 9 pages (electronic).

[3] P. Pollack, 'On Dickson's theorem concerning odd perfect numbers', Amer. Math. Monthly 118 (2011), 161-164.

[4] P. Yuan, 'An upper bound for the number of odd multiperfect numbers', Bull. Aust. Math. Soc. 89 (2014), 1-4.

PINGZHI YUAN, School of Mathematics, South China Normal University, Guangzhou 510631, PR China

e-mail: yuanpz@scnu.edu.cn

ZHONGFENG ZHANG, School of Mathematics and Informational Science, Zhaoqing University, Zhaoqing, 526061, PR China

e-mail: zh12zh31f@yahoo.com.cn 